\title{
Targeted therapies in cancer and mechanisms of resistance
}

\author{
Simona Corso • Silvia Giordano
}

Published online: 4 June 2014

(C) Springer-Verlag Berlin Heidelberg 2014

Targeted therapies by means of compounds that inhibit a specific target molecule represent a new perspective in the treatment of cancer. In contrast to conventional chemotherapy, which acts on all dividing cells, targeted drugs allow to hit subpopulations of cells directly involved in tumor progression in a more specific manner [1].

The frequent alteration of receptor tyrosine kinases (RTK) in human malignancies led them to be considered as targets for anti-neoplastic therapies; this resulted in the development of several inhibitors that have shown a strong clinical activity [2]. The concept of "oncogene addiction" has added further rationale to the use of targeted therapies [3]. Even if the recent introduction in cancer therapy of several selective tyrosine kinase inhibitors has had a dramatic effect in oncology, after the first excitement following the initial results, the clinicians had to face soon the problem of primary and secondary resistance to treatment: a percentage of patients expressing the target in their tumors does not respond to the treatment (primary or "de novo" resistance), while in most of the responders, the treatment is quite rapidly no longer effective (secondary or "acquired" resistance) (Fig. 1) [4]. Therefore currently, the main challenges associated to targeted therapies are the relatively small proportion of patients that can benefit from these treatments and the almost inevitable occurrence of resistance. Thus, the identification of predictive biomarkers of resistance and the understanding of the resistance mechanisms are mandatory to improve the efficacy of these therapies.

S. Corso $\cdot$ S. Giordano $(\bowtie)$

Department of Oncology, University of Torino,

Strada Provinciale 142, Candiolo, Torino 10060, Italy

e-mail: silvia.giordano@ircc.it

S. Corso $\cdot$ S. Giordano

Candiolo Cancer Institute-FPOIRCCS, Strada Provinciale

142, Candiolo, Torino 10060, Italy
Genetic studies have recently highlighted the high level of heterogeneity in tumors, where many clones with different genetic features coexist. Cancers, in fact, evolve through a series of waves of clonal growth where single clones acquire genetic alterations conferring differential fitness [5]. Moreover, environmental factors (such as secretion of cytokines by the stromal cells or the availability of nutrients and oxygen) can favor the onset and permanence of such different cell populations. In this scenario, exposing a tumor to a targeted therapy can abruptly subvert the equilibrium that allows the coexistence of these cell populations. A paradigmatic example is represented by lung cancers displaying activating epidermal growth factor receptor (EGFR) mutations that render them responsive to EGFR inhibitors. Treatment with EGFR inhibitors, in fact, results in massive death of EGFR-addicted cancer cells, thus profoundly impacting on the overall architecture of the tumor. Cells that are not responsive to the inhibitor (such as those harboring the "resistant" EGFR mutation T790M) preexist inside the tumor and can outgrow under the selective pressure imposed by the drug $[6,7]$. So, a cell population present in the primary cancer at low frequency due to a decreased fitness compared to cells devoid of the mutation becomes enriched over time and can reconstitute the tumor, leading to relapse (Fig. 1). Thus, from the beginning, the tumor contains cells harboring genetic lesions that can sustain resistance to targeted therapies. As mentioned by Dr. Vogelstein in a recent paper, "resistance is a fait accompli and the time to recurrence is simply the interval required for the subclone to repopulate the lesion" [8]. The presence of genetic heterogeneity in the primary tumor may also explain the common finding of patients presenting, at relapse, different metastases, each bearing a diverse genetic alteration responsible for drug resistance. The knowledge of the existence in primary tumors of cell subpopulations harboring genetic alterations leading to drug resistance has induced researchers and clinicians to test the efficacy of combination therapies in 
Fig. 1 In tumors showing primary resistance, the majority of neoplastic cells do not respond to the treatment, although bearing the drug target. This can be due to the activation of downstream intracellular effectors (as shown in the figure). Resistant tumors can also contain cell subpopulations in which resistance is due to different mechanisms. In tumor displaying secondary resistance (the relapse after an initial response), the vast majority of tumor cells expresses the drug target and responds to the treatment, leading to shrinkage of the tumor mass. However, minor cellular subpopulations may be unresponsive, for example because of lack of target, activation of alternative parallel pathways, or activation of downstream effectors. In principle, each of these cells can repopulate the tumor, leading to relapse. Therefore, relapses at different sites can display different mechanisms of resistance
PRIMARY RESISTANCE

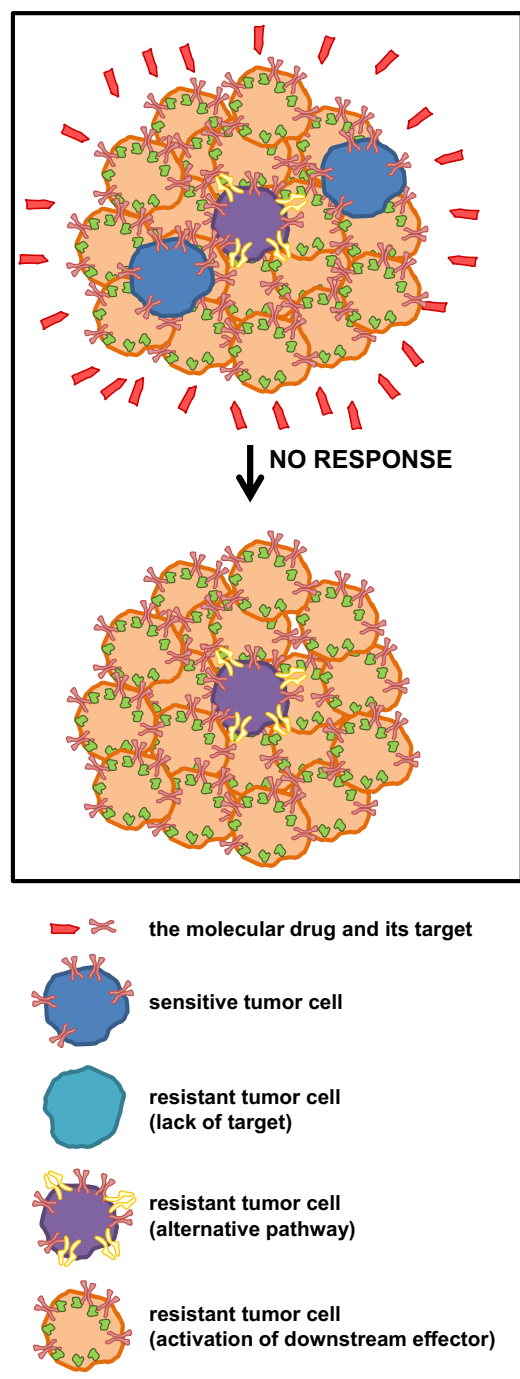

SECONDARY ('ACQUIRED') RESISTANCE
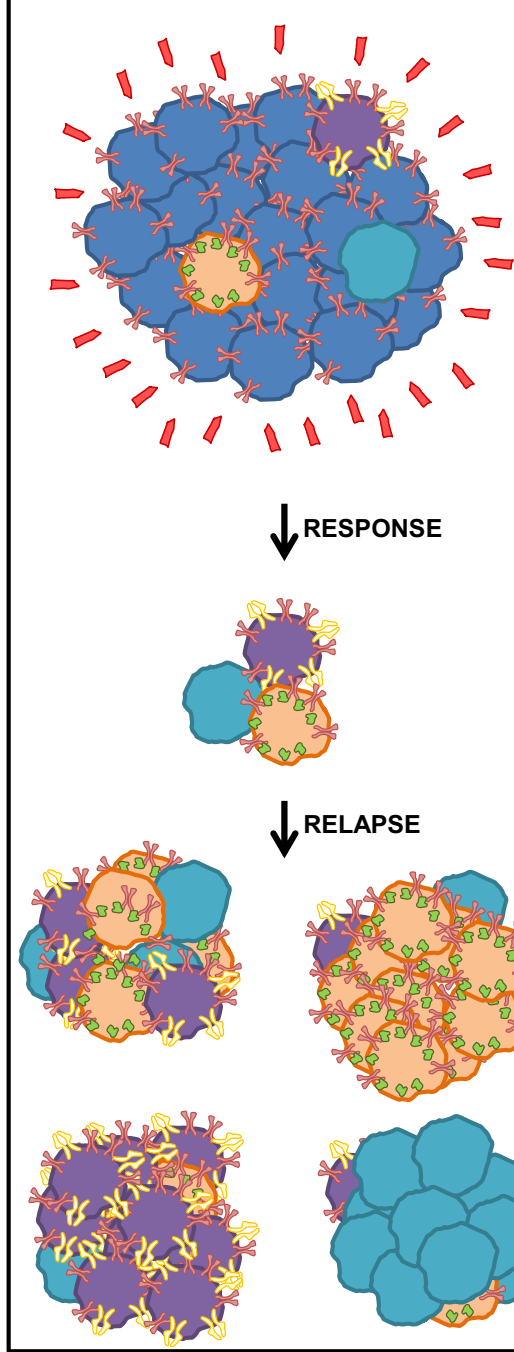
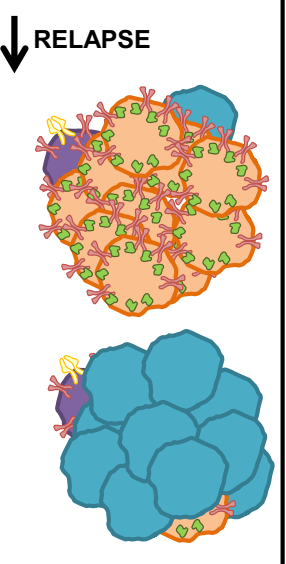

preclinical and clinical settings. Clinical trials that combine drugs hitting different targets are now ongoing, and their results will tell us the effectiveness of this strategy.

Another critical problem dealing with sensitivity/resistance to targeted therapies is the identification of biomarkers. Personalized medicine aims at targeting driver lesions in the tumors where they are present and that are addicted to them. Even if the occurrence of these alterations is a predictive biomarker, their presence is not sufficient to guarantee a response to treatment. An emblematic example is given by the BRAF V600E mutation that occurs in melanomas and colon carcinomas. While treatment with vemurafenib (targeting mutated BRAF) results in $80 \%$ response rate in melanoma, it is very rarely effective in colon carcinoma [9]. The presence of the altered driver is, thus, necessary but not sufficient to guarantee a response, and other biomarkers are required to identify exactly the responsive patients. In some cases, responsive patients can be better detected using negative biomarkers. This is the case of colon cancer where the presence of Kirsten rat sarcoma viral oncogene homolog (KRAS) mutations predicts the lack of response to the antiEGFR antibodies cetuximab and panitumumab [10,11].

Although conceptual progress has been made to better understand the mechanisms underlying primary and secondary resistance, many challenges still remain in the field of targeted therapies. Some of them are discussed in the four reviews presented in this special issue.

Fabi and colleagues present the cutting-edge therapies targeting HER2 in breast cancer [12]. Together with antiestrogen treatments, HER2 inhibition represents the first example of an effective targeted therapy in solid tumors. Trastuzumab has been approved in 1998 for HER2 overexpressing metastatic breast cancer, and its use has been further extended in 2006 to women with node-positive, HER2overexpressing breast cancer, in association with chemotherapy. The recent approval of other HER2 targeting drugs (pertuzumab, lapatinib, and T-DM1) has opened new opportunities, together with new problems: which is the best drug? 
Which is the best combination? Which is the best schedule of administration? Can resistance to one of these drugs be overcome by the use of any of the others? Fabi and colleagues discuss these problems based on available data and elaborate the outstanding clinical issues related to HER2 therapeutic targeting.

Gower and colleagues review the progress made by targeted therapies in lung adenocarcinomas [13]. Over the past few years, molecular studies revealed that lung cancer comprises many subtypes and may serve as a prototype for a biology-based tumor reclassification that combines morphologic and genomic data. On these premises, lung cancer treatment has become the prototype for genetically tailored targeted therapies. Gower and colleagues discuss approved therapies, such as those targeting EGFR and ALK, as well as the new ones directed against recently discovered drivers (ROS1, RET, MET, HER2, BRAF and PI3K).

Leto and Trusolino discuss the mechanisms of primary and acquired resistance to EGFR-targeted therapies in colorectal cancer [14]. In this tumor type, there is no predictor of response to EGFR antibodies, but clinical treatment takes advantage of the identification of several negative predictors of response, such as mutations of KRAS, BRAF, or amplification of either MET or HER2. However, there are nonresponding patients that do not display any of these alterations but are resistant to treatment. The identification of new biomarkers to find actionable drivers is, thus, a critical point for the future treatment of this tumor.

Finally, in their review, Carlomagno and Chiariello summarize the recent findings in preclinical and clinical studies performed by blocking the RAS/MAPK pathway in anti-cancer therapy with a specific focus on melanoma, and discuss novel therapeutic approaches such as the use of anti-RAS inhibitors [15]. The knowledge acquired about this pathway as a result of remarkable efforts of basic and translational research has improved our comprehension of unpredicted molecular mechanisms underlying the pathogenesis of cancer and has opened new perspective to target these molecules.

\section{References}

1. Chabner BA, Roberts TG (2005) Timeline: chemotherapy and the war on cancer. Nat Rev Cancer 5:65-72

2. Gschwind A, Fischer OM, Ullrich A (2004) The discovery of receptor tyrosine kinases: targets for cancer therapy. Nat Rev Cancer 4:361-370

3. Weinstein IB, Joe AK (2006) Mechanisms of disease: oncogene addiction - a rationale for molecular targeting in cancer therapy. Nat Clin Pract Oncol 3:448-457

4. Holohan C, Van Schaeybroeck S, Longley DB, Johnston PG (2013) Cancer drug resistance: an evolving paradigm. Nat Rev Cancer 13: 714-726

5. Bedard PL, Hansen AR, Ratain MJ, Siu LL (2013) Tumour heterogeneity in the clinic. Nature 501:355-364

6. Turke AB, Zejnullahu K, Wu YL, Song Y, Dias-Santagata D, Lifshits E, Toschi L, Rogers A, Mok T, Sequist L et al (2010) Preexistence and clonal selection of MET amplification in EGFR mutant NSCLC. Cancer Cell 17:77-88

7. Maheswaran S, Sequist LV, Nagrath S, Ulkus L, Brannigan B, Collura CV, Inserra E, Diederichs S, Iafrate AJ, Bell DW et al (2008) Detection of mutations in EGFR in circulating lung-cancer cells. N Engl J Med 359:366-377

8. Diaz LA, Williams RT, Wu J, Kinde I, Hecht JR, Berlin J, Allen B, Bozic I, Reiter JG, Nowak MA et al (2012) The molecular evolution of acquired resistance to targeted EGFR blockade in colorectal cancers. Nature 486:537-540

9. Prahallad A, Sun C, Huang S, Di Nicolantonio F, Salazar R, Zecchin D, Beijersbergen RL, Bardelli A, Bernards R (2012) Unresponsiveness of colon cancer to BRAF(V600E) inhibition through feedback activation of EGFR. Nature 483:100-103

10. Amado RG, Wolf M, Peeters M, Van Cutsem E, Siena S, Freeman DJ, Juan T, Sikorski R, Suggs S, Radinsky R et al (2008) Wild-type KRAS is required for panitumumab efficacy in patients with metastatic colorectal cancer. J Clin Oncol 26:1626-1634

11. Karapetis CS, Khambata-Ford S, Jonker DJ, O'Callaghan CJ, Tu D, Tebbutt NC, Simes RJ, Chalchal H, Shapiro JD, Robitaille S et al (2008) K-ras mutations and benefit from cetuximab in advanced colorectal cancer. N Engl J Med 359:1757-1765

12. Fabi A, Mottolese M, Segatto O (2014) Therapeutic targeting of ERBB2 in breast cancer: understanding resistance in the laboratory and combating it in the clinic. J Mol Med doi:10.1007/s00109-014-1169-7

13. Gower A, Wang Y, Giaccone G (2014) Oncogenic drivers, targeted therapies, and acquired resistance in non-small-cell lung cancer. $\mathrm{J}$ Mol Med doi:10.1007/s00109-014-1165-y

14. Leto SM, Trusolino L (2014) Primary and acquired resistance to EGFR-targeted therapies in colorectal cancer: impact on future treatment strategies. J Mol Med doi:10.1007/s00109-014-1161-2

15. Carlomagno F, Chiariello M (2014) Growth factor transduction pathways: paradigm of antineoplastic targeted therapy. J Mol Med doi:10. 1007/s00109-014-1177-7 